\title{
Le Clown dans l’Enseignement de Jacques Lecoq
}

\author{
Guy Freixe \\ Professeur de l'Histoire etd'esthétique des Arts de la Scèneà l'Université Franche-Comté, Besançon, France. \\ Comédien, metteur en scène et pédagogue de théâtre formé à l'École Jacques Lecoq. \\ guy.freixe@gmail.com
}

Résumé

Le présent article nous montre que la pratique du style clown développé à l'École Internationale de Mime,Théâtre et Mouvement Jacques Lecoq appartient à la tradition de l'École du Vieux Colombier créé par Jacques Copeau dont le fondement est lesprit enfantin et le jeu.

Palavras-chave

Clown. Jacques Lecoq. Jacques Copeau.
Resumo

O presente artigo nos mostra que a prática do estilo clown tal como é desenvolvida na Escola Internacional de Mimo, Teatro e Movimento de Jacques Lecoq pertence à tradição da Escola do Vieux Colombier criada por Jacques Copeau, que tem como base o espírito infantil e o jogo.

Mots clés

Clown. Jacques Lecoq. Jacques Copeau. 
Le renouveau du clown de théâtre doit beaucoup à l'enseignement de Jacques Lecoq. En 1962, six ans après la création de son école à Paris, Lecoq commence un travail sur le jeu clownesque qui s'avèrera déterminant pour le renouveau du clown; un clown qui quittera la piste du cirque où il se meurt pour gagner la rue, les cabarets, les scènes de théâtre. Lecoq a œuvré à cette mutation du clown de cirque vers le clown de théâtre, ouvert à des situations dramatiques renouvelées. Territoire dramatique qu'il nommera dans son enseignement, à partir des années 80 , les « variétés comiques ", incluant le burlesque, l'absurde, les excentriques...

Mais avant de s'intéresser à cet enseignement, faisons un petit détour pour mieux comprendre I'héritage reçu par Lecoq, et éclairer ainsi sa filiation avec Jacques Copeau'1.

Le « vrai acteur»: le clown

Copeau a été un semeur de graines. Un initiateur et un éveilleur. II a toujours pensé avant même de fonder, il y a de cela tout juste cent ans, le Théâtre du Vieux-Colombier - que la rénovation urgente du théâtre devait passer en premier par l'acteur. Et qu'il fallait débarrasser la scène et le jeu des tricheries et faussetés accumulées depuis des lustres. C'est du côté de l'enfance, de l'instinct de jeu de l'enfant, que Copeau va chercher les fondements de sa pédagogie. Avait-il en tête l'aphorisme de Baudelaire : «Le génie, c'est l'enfance retrouvée à volonté " ? Le fait est que Copeau attachait la plus grande importance au jeu des enfants

1 Cf. Freixe G., La Filiation Copeau - Lecoq - Mnouchkine, une lignée du jeu de l'acteur, Montpellier, L'Entretemps, coll. « Les voies de l'acteur », 2014. et avait jugé bon de ne pas envoyer les siens à l'école, comme nous l'apprend sa fille Marie-Hélène Dasté, afin qu'ils tirent de leurs jeux seuls toute leur instruction :

"Nous étions habités par une espèce d'invention perpétuelle, encouragée par mon père, et qui d'ailleurs pour lui était comme la loi, le secret de l'éducation d'un acteur: lui faire garder son esprit d'enfance, lui faire retrouver toujours cette faculté d'émerveillement et d'étonnement qui est celle des enfants ${ }^{2}$. 》

Copeau voulait préserver chez l'acteur cette faculté de poésie et de créativité, cet état de jeu fait de disponibilité, d'invention, d'attention et d'imagination, et c'est avec de jeunes enfants de dix à quatorze ans qu'il va commencer, pendant la guerre, à mener ses premiers exercices pédagogiques pour se préparer à l'ouverture de son École du Vieux-Colombier. En proposant un travail autour des fables de La Fontaine, il se montre dès le début vigilant à ce que les enfants entrent dans les personnages par le corps : non pas seulement en « signifiant » l'animal pour qu'il soit reconnaissable, mais en le « mimant intérieurement », en recherchant par exemple « la démarche silencieuse et déliée du chat pour qu'apparaisse son caractère ${ }^{3}$... Copeau veut que ce soit par le corps et une imagination toute physique que l'enfant se lance en premier dans le jeu dramatique. II se méfie des mots et de l'analyse psychologique des personnages de la fable, comme si

2 Rencontre avec Marie-Hélène Dasté, Catherine Dasté, Christophe Allwright : une famille d'acteurs, rencontre organisée le 19 mars 1993 dans le cade des Ateliers de formation permanente du Théâtre du Campagnol, éd. Théâtre du Campagnol - Centre Dramatique National de Corbeil-Essonnes, VHS.

3 Copeau J., Registres VI : L'École du Vieux-Colombier, textes établis, présentés et annotés par Sicard $\mathrm{Cl}$., Paris, Gallimard NRF, coll. " Pratiques du théâtre », 2000, p. 99. 
la rationalité du langage éloignait l'enfant du mimétisme premier et de la spontanéité créatrice. Copeau se dit enchanté par ces premiers exercices sur l'improvisation à partir des animaux et il est conscient qu'ils vont orienter sa recherche future : « Cette mine d'observation, écrit-il dans son Cahier, et l'expérience de ces exercices seront d'un grand secours à l'artiste pour la représentation plus ou moins forcée et caricaturée des personnages de la comédie ${ }^{4}$.» Copeau cherche à déclencher chez l'enfant un jeu libre, inventif, personnel, qui le "transporte" dans l'imaginaire. II attachera à ce mouvement libératoire la plus grande importance par la suite. Mais pour cela, il faut que l'enfant entre totalement dans son jeu, qu'il joue vraiment, c'est-à-dire qu'il quitte la réalité pour vivre dans l'imaginaire. C'est la raison pour laquelle il refuse toute inféodation à l'écrit : «Se défier surtout de ce qui nous rapproche de la littérature. Fuir aux antipodes. Pas de rapport ni de communication. II ne s'agit pas d'improviser d'après, mais d'improviser tout court. Pas d'intermédiaire. L'improvisateur vaut ce qu'il vaut. Et tant vaut l'improvisation... ${ }^{5}$. » Copeau se rend compte alors qu'il y a deux écoles possibles, qui s'opposent quant à la nature du jeu recherché : l'école de l'improvisation et l'école de l'interprétation. Dans l'improvisation, l'acteur est confronté avant tout au présent et à l'instant partagé avec le partenaire et le public. Dans l'interprétation, qui part du texte dramatique, il doit médiatiser avec l'épaisseur sémantique du langage. Copeau opte pour l'improvisation. II s'en ouvre rapidement à ses deux complices, Charles Dullin et Louis Jouvet, qui sont au front. Leur correspondance,

4 ldem, p. 101.

5 Note de Copeau sur la lettre de Jouvet du 10 janvier 1916, in Registres VI : L'École du Vieux-Colombier, op. cit., p. 110. de décembre 1915 à avril 1916, tourne autour de cette formidable révolution qu'est la découverte du jeu improvisé et des perspectives nouvelles qu'elle ouvre.

En janvier 1916, Copeau franchit une nouvelle étape. II découvre avec enthousiasme les improvisations clownesques des trois frères Fratellini. II écrit à sa femme : « Je crois que je n'aime plus réellement que la farce au théâtre. J'ai été cinq fois au Cirque revoir les mêmes clowns ${ }^{6}$. " II admire leurs lazzi, et leur jeu sans cesse renouvelé avec le public : «Un soir, un de ses lazzi déclenche le rire sonore et prolongé d'une dame de la galerie. [Le clown] s'arrêta net et, campé de profil, dévisagea la rieuse, sans bouger, les sourcils hauts. Et comme la femme riait de plus en plus, il ne cessait de la regarder. Puis il reprit son exercice, et il ne fit rien, ce soir-là, qui ne fût en fonction de cette complicité qu'il avait dans la salle. La saveur de son jeu en était décuplée ${ }^{7}$. » II admire « la grâce alerte de leurs corps entraînés ${ }^{8}$ », qui leur permet de trouver une transposition des sentiments par une écriture poétique du geste, et leur capacité à inventer au présent, dans une interaction constante avec la salle. Il est frappé par la justesse de la définition d'un des principes de leur art : "Le mouvement, le rythme et la précision ${ }^{9}$. " Copeau rapporte dans son carnet ce qu'il écrit, en juin de la même année, à son ami Jaques Dalcroze : « (...) Et puis voir se replacer, en serviteur de livrée, au cirque, celui (l'Individualité précise) que l'on vient de

6 Copeau J., Cahier "La Comédie improvisée », Registres III : Les Registres du Vieux Colombier I, textes recueillis et établis par Dasté M. H. et Maistre Saint-Denis S., Paris, Gallimard NRF, coll. " Pratique du théâtre », 1979, p. 319.

7 Idem., p. 320.

$8 \mathrm{lbid}$.

9 lbid. 
voir faire des miracles... - j'allais au cirque pour cela ! L'un se détache du groupe - puis revient - pour un numéro sensationnel - puis ressort et rentre - en livrée - dans la Communauté. C'est beau ! - Voilà le vrai acteur ${ }^{10}$ ! »

Copeau écrit à son ami André Gide : « L'aire vide de la piste hante mes insomnies ${ }^{11}$. " Cet espace de tous les possibles deviendra pour lui l'espace de jeu idéal : une scène désencombrée, peuplée entièrement par l'imagination de l'acteur. II n'oubliera jamais qu'un clown porte avec lui l'espace de jeu qui lui est nécessaire. Son costume seul est son propre décor. II y a là pour Copeau une leçon essentielle qui relie le clown à la vitalité d'une tradition, à cet acteur improvisateur de la commedia dell'arte qui savait puiser dans l'enfance la force première de sa fantaisie.

Au vide du plateau, Copeau veut accorder le vide intérieur de l'acteur. Un vide qui fasse vibrer l'être en le reliant à l'authenticité de son engagement. Un vide mystérieusement plein. C'est ce vide que Copeau recherchait justement à faire naître dans ses premiers essais d'improvisation avec les enfants, en suivant le développement naturel de leur instinct de jeu, en les encourageant et les accompagnant dans leur chevauchée imaginaire. Ce travail avec les enfants, et ses réflexions liées au jeu improvisé des clowns, ont radicalement changé sa manière de travailler. Désormais, Copeau renonce à emplir l'acteur d'informations et d'explications sur le jeu et le caractère du personnage. II préfère faire appel à la puissance mystérieuse de ce vide pour que les ailes de l'imagination de l'acteur se déploient, et qu'il se laisse porter jusqu'à l'incarnation de son personnage.

10 Idem., p.321.

11 Idem, p. 322.
Du clown de cirque au

clown de théâtre : la pédagogie du « bide »

Jacques Lecoq, qui ouvre son École à Paris en 1956, est dans la continuité pédagogique de- l'École du Vieux-Colombier de Copeau (1921-1924). II vise avant tout à éveiller l'instinct dramatique de ses élèves en les faisant improviser dans le silence d'avant les mots. Le corps y est l'instrument premier de l'acteur. Le masque un positionnement initial visant au retrait de la subjectivité : l'acteur tend à se vider de lui-même, à devenir impersonnel pour mieux recevoir les phénomènes du vivant et plus tard les réfracter dans son jeu. Dans cette lignée de théâtre, ce qui « apparaît » dans les manifestations corporelles de l'acteur importe plus que le « dissimulé ». Au début de son enseignement, Lecoq s'appuie essentiellement sur l'étude des grands territoires dramatiques que sont la tragédie grecque, avec la dynamique du chœur et du héros, et la commedia dell'arte, qu'il contribua avec Amleto Sartori et Giorgio Strehler à « ressusciter » en collaborant au mythique Arlequin serviteur de deux maitres de Goldoni. Ce n'est qu'à partir de 1962 qu'il fera une incursion du côté du jeu clownesque, qui deviendra vite un temps fort de son enseignement. Et cela en s'interrogeant sur la nature de la relation entre la commedia dell'arte et les clowns de cirque. Lecoq cherchait par des exercices à mieux saisir l'émergence du rire :

« J'installais la piste et chacun s'y présentait avec la seule obligation de nous faire rire. C'était terrible, ridicule, personne ne riait ; les élèves-clowns prenaient le « bide » dans l'angoisse générale ; et, à mesure que chacun passait, le même phénomène se renouvelait. Le clown dépité allait s'asseoir, penaud... et c'est à ce momen- 
t-là que nous commencions à rire de lui. La pédagogie était trouvée, celle du « bide ${ }^{12}$. »

De quoi riait-on ? Non du personnage que les élèves tentaient de présenter, mais de la personne elle-même, mise à nu. Lecoq se rend compte alors de la spécificité du clown, éloigné de tous les autres registres et territoires de jeu où il s'agit au fond de jouer quelqu'un d'autre que soi. Le clown est bien différend, car il n'existe pas en dehors de l'acteur qui le joue. II n'y a pas de doublure à un clown. À la différence de la commedia dell'arte, où l'acteur passe d'un masque à un autre, changeant d'énergie et de corps pour incarner tour à tour Arlequin, Pantalon ou le Capitan, il n'a pas avec le clown à entrer dans un personnage préétabli. Il doit découvrir en lui la part clownesque qui l'habite. Moins l'acteur se défend, moins il essaie de jouer un personnage, plus il se laisse surprendre par ses propres faiblesses, plus son clown aura des chances d'apparaître...

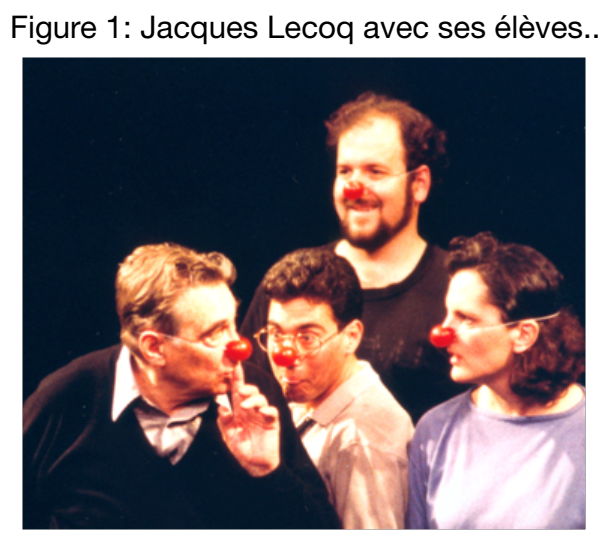

Le petit nez rouge du clown, cette boule de couleur qui illumine les yeux et arrondit le visage, agit comme un masque, "le plus petit masque du monde "selon l'expression de Lecoq. Car, comme un masque, le nez rouge opère une mutation. Quand on le met, ce n'est

12 Lecoq J., Le Théâtre du geste (sous la direction de), entretien avec Perret J., Paris, Bordas, 1987, p. 116. pas seulement un simple objet que l'on pose sur le visage, c'est un événement qui survient. L'intime qu'il s'agit de sortir de soi prend tout à coup une force nouvelle. On se sent plus large, disponible, capable de transformer ses faiblesses en force théâtrale, ses ratages en triomphe. À force de louper, d'être nul, de multiplier les échecs, le clown à un moment où il ne l'attend pas se surprend et nous surprend par sa virtuosité... car il y a toujours en lui, enfouies, cachées - comme en chacun de nous - des prouesses inédites, et derrière sa balourdise apparente, des trésors d'adresse insoupçonnés, le clown surprend toujours et vient démystifier la prétention que nous avons lorsque nous nous croyons supérieur aux autres, car il nous rend sensible à la beauté dans l'inadaptation même au réel.

Le postulat de la pédagogie du clown de Lecoq est un peu le suivant : nous sommes tous des clowns, dans le sens où nous nous voulons tous beaux, intelligents, forts... alors que nous avons chacun nos faiblesses, nos zones de fragilité, notre « dérisoire ${ }^{13}$ »... et c'est cela qui, en s'exprimant, en sortant au grand jour, fait rire. Cette découverte de la transformation d'une faiblesse personnelle (physique ou psychique) en force théâtrale fut de la plus grande importance pour Lecoq. Cela lui permit de mettre au point une approche pédagogique personnalisée de cette " recherche de son propre clown ", devenue une étape essentielle de son enseignement et le temps fort, après deux années, de la fin du voyage de l'École.

13 « La recherche de son propre clown, c'est d'abord la recherche de son propre dérisoire. ", in Lecoq. J, en collaboration avec Carasso J-G., Lallias J-Cl., Le Corps poétique, Arles, Actes Sud-Papiers/ANRAT, 1999, p. 154. 
Du masque neutre au clown : la géométrie inversée

La pédagogie de Lecoq est encadrée par deux grands moments de recherche : le masque neutre, qui occupe six mois de la première année, et le clown, en fin de deuxième année, qui est une ouverture à l'après-école. D'un côté, le masque où l'on se quitte, pour accueillir en soi les grands rythmes du monde du vivant, et où l'on tend tous vers un même dénominateur commun ; de l'autre, "le plus petit masque du monde " qui nous fait exister comme sujet et où l'individu apparaît dans la singularité de son propre dérisoire. Mais un dérisoire qui appartient à tout le monde, et définit poétiquement la condition humaine...

Le masque neutre est une référence commune, quelque peu abstraite, qui vise à rassembler et unifier les élèves. II est ce commun dénominateur qui nous unit pour aller à la recherche des fondamentaux de la vie. Sous ce masque, l'élève accomplit un " voyage élémentaire " qui nous fait éprouver les grands rythmes de notre destinée commune. Sous ce masque, l'élève s'identifie aux dynamiques des éléments : eau, vent, terre, feu ; aux matières : bois, papier, métal, soie, verre, caoutchouc...; aux couleurs et aux sons. Lecoq recherche l'imprégnation corporelle plus que l'expression corporelle. Le rythme de la forêt, la lumière solaire, la montagne que l'on gravit, la pente que l'on descend pour regarder le crépuscule dans la plaine... l'élève doit d'abord en retrouver l'impression dans son corps, avant d'en donner une expression. Le masque aide à cette dépersonnalisation pour que le corps puisse nous faire retrouver concrètement le souffle du vent, le mouvement des vagues, l'enracinement de l'arbre et la folie dansante du feu. L'identifica- tion « mimo-dynamique » permet ainsi de faire ressurgir “ des gestes oubliés ${ }^{14}$ », et ouvre à ce qui nous restait autrement impénétrable. L'acteur retrouve alors ce que le corps a gardé en mémoire de toutes ses expériences sensibles, et c'est de ces strates enfouies que naissent les élans profonds de l'expression. Lecoq a fait de ce postulat le fondement de sa démarche pédagogique et créatrice : « La nature est notre premier langage. Et le corps se souvient ${ }^{15}$ ! »

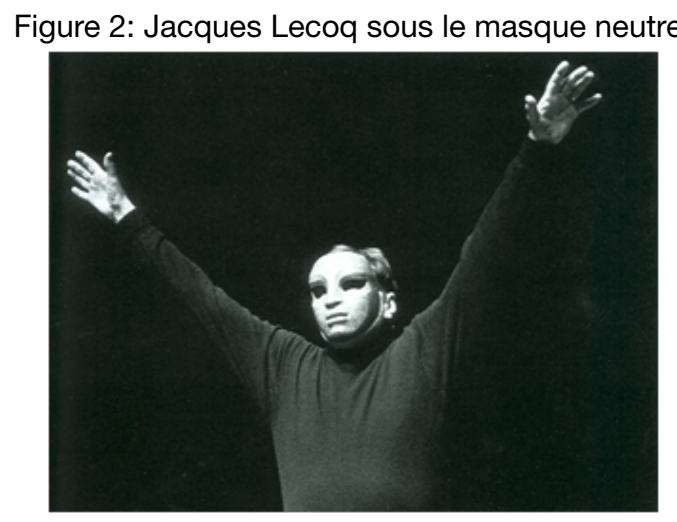

Figure 3: Emma la clown (Meriem Menant, qui a découvert son clown à l'École Lecoq).

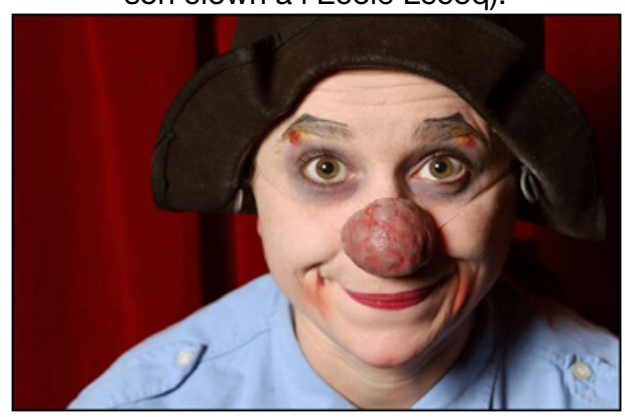

Avec le clown, Lecoq touche la limite inverse de son enseignement : alors que pendant deux années l'élève part du monde extérieur pour le laisser se refléter en lui, il lui demande à la fin de son parcours d'apprentissage, paradoxalement, d'être le plus simplement et profondé-

14 Cf. Skansberg C., « La recherche des gestes oubliés. L'École de Jacques Lecoq ", La Formation du comédien, études réunies et présentées par Gordon A. M., Paris, éd. CNRS, coll. "Les Voies de la création théâtrale », T. IX, 1981, p. 7991.

15 Lecoq J, Le Corps poétique, op. cit., p. 56. 
ment possible lui-même, et d'observer l'effet qu'il produit sur le monde, c'est-à-dire sur le public. Car le clown n'existe qu'à partir du public. II garde toujours un contact direct et immédiat avec lui. II ne peut vivre qu'avec et sous son regard : "On ne fait pas le clown devant un public, on joue avec lui ${ }^{16}$. " Lecoq a comme premier exercice la simple présentation du clown devant le public... pour que l'élève apprenne à se « poser » sur lui, prêt à réagir à son contact, comme si le petit nez rouge était en équilibre sur le jet d'eau d'une fontaine. On reste ainsi totalement disponible à l'événement du public. Emma la clown, ou plutôt Meriem Menant ${ }^{17}$, m'a confié que cet exercice de Lecoq avait été pour elle fondamental, et qu'aujourd'hui encore elle adore retrouver cette sensation en s'arrêtant tout à coup dans son spectacle-solo pour attendre et recevoir ainsi l'impulsion du public : «Ce qui est fou, c'est que je ne sais jamais combien de temps cet arrêt va durer. Je ne fais plus rien. Rien. Le temps alors n'a plus de prise. C'est une sensation vertigineuse, enivrante, dangereuse aussi. Mais j'adore ça ${ }^{18}$. » Moment périlleux de totale disponibilité au présent, où l'on ne sait plus ce qui va advenir. Comme si on était porté et soulevé par le corps collectif de la salle... Vide matriciel de l'acteur qui nous fait penser à ce que nous évoquions plus haut au sujet de la fascination de Copeau pour le clown et sa scène vide.

Autre élément paradoxal dans la pédagogie de Lecoq : alors que l'attention a toujours été

\section{Idem., p. 156.}

17 Meriem Menant a suivi les deux années de formation de l'École Jacques Lecoq où son clown est né. Elle a créé plusieurs solos, dont Emma la clown sous le divan (2004), Dieu est-elle une particule ? (2009), Emma Mort même pas peur (2013), et tourne depuis de nombreuses années avec Catherine Dolto La Conférence, et Grand Symposium : tout sur l'amour.

18 Interview de Meriem Menant par l'auteur, 28 janvier 2014. portée sur le collectif, la dimension chorale du jeu, la création en groupe... le clown est profondément seul. Sa solitude, son unicité fait sa force. Il conduit l'élève vers le singulier, l'intime, le subjectif, l'irrationnel, le surgissement du pulsionnel, tout ce qui avait été jusqu'alors tenu à l'écart de l'exploration dramatique. Mais ce clown surgit avec d'autant plus de force qu'il a été préparé par un mouvement contraire : nulle psychologie ni complaisance à soi dans cette recherche du clown, car le masque du nez rouge condense tout le travail mené à l'École sur le rythme, le mouvement, les passions, l'écriture poétique du corps. Ainsi, l'intime mobilisé et sollicité trouve une force nouvelle, une cristallisation dramatique.

\section{Le surgissement du clown}

Le clown n'a besoin de presque rien pour vivre et improviser en scène : une valise, une chaise, un chapeau... l'exceptionnel doit venir du très banal en somme.

Le personnage a besoin de conflits avec le monde extérieur. Pas le clown, car il est en permanence en conflit avec lui-même. Est-ce d'ailleurs un personnage cette créature surgie du fond de soi ? Comment la faire advenir d'ailleurs ? Lecoq avance ici, comme toujours, d'une manière très concrète: il demande par exemple à l'élève de montrer une chose qu'il est le seul à réussir, de jouer d'un instrument de musique, de se costumer dans la plus grande liberté, en gardant en tout l'esprit d'enfance.

Meriem Menant se souvient d'avoir trouvé son clown immédiatement, en revêtant ces mêmes habits de cheftaine scout qu'elle continue de porter depuis 25 ans. Elle ne sait pas bien comment cela est arrivé, mais alors qu'elle 
avait eu beaucoup de mal à l'École avec les exercices du masque neutre « qui l'ennuyaient profondément », et qu'elle « grimaçait » le plus souvent en improvisation, soudain, en clown, elle s'est sentie libre, tellement libre, car elle n'était plus du tout Meriem mais une « créature » qui savait tout avant elle, et qui possédait un aplomb sidérant ${ }^{19}$. Ce clivage est d'ailleurs toujours très fort entre son clown et elle, entre Emma et Meriem... Catherine Dolto - sa partenaire de la fameuse Conférence - s'en explique :

«Oser parler de la relation entre un clown et celui qui le porte sur scène, son porteur, est d'une grande audace [...]. En savoir trop pourrait menacer l'alchimie merveilleuse qui permet au clown de continuer à vivre et à tirer son porteur en scène à travers lui. On pourrait dire son auteur, mais un clown ça se porte, en soi et hors de soi ${ }^{20}$. ”

En scène, Meriem nous l'a dit, elle ne sait pas qui s'exprime... Quand Emma est sur scène, Meriem est ailleurs... mais où ? Partout sans doute, diffractée en milliers de petits fragments cachés en Emma, mais de telle manière qu'ils ne peuvent se regrouper pour dire « je ». C'est le clown qui dit « je », et Meriem l'écoute, sans forcément se reconnaître. Sans être dupe pour autant. Ce n'est pourtant pas « elle », ce sont « elles » qui parlent et agissent en scène.

II y a entre le clown et son porteur un mystère... L'acteur peut « incarner » divers personnages, mais quand un clown surgit et s'empare de lui, il reste unique. II n'y a pas « un de mes clowns », mais «mon clown ${ }^{21 » . ~ C e ~ c l o w n ~ e s t ~}$

19 Cf. notre entretien avec Meriem Menant.

20 Dolto C., Memma et Eriem Qui est qui ? Le clown et son porteur, 20 septembre 2009, texte inédit communiqué par Meriem Menant,

21 Cf. « Nous pouvons à plusieurs acteurs jouer le même personnage, mais pas le même clown. Sans doute parce qu'un clown ne se joue pas. II se respire. ", artigo de Catherine Ger- une présence énigmatique par delà toutes les distinctions identitaires de sexe, ou d'âge... Ce n'est pas un double non plus... mais la manifestation de l'ailleurs, l'essence de l'être, le « poème » de la présence, comme l'écrit François Cervantes:

« Le clown ne dit pas un poème, il ne fait pas un poème, il "est" un poème. II est avec son corps comme l'auteur est avec le langage.

Pour lui, le corps, cet enchevêtrement de muscles de nerfs et de peau, c'est le langage, et mettre à jour le clown, c'est mettre à jour le poème incarné, la présence unique de ce corps, rendre lisible le poème écrit par la vie, inscrit dans le grand livre ${ }^{22}$. 》

II y a quelque chose de magique quand le clown entre et sort de scène. Comme s'il n'était pas foncièrement d'ici... Les clowns sont des êtres à peine incarnés. Cette extravagance dans laquelle on les voit apparaître, c'est comme s'ils étaient un corps à nu qui ne sait pas encore qui il est, qui il pourrait être, quelle place il pourrait avoir dans le monde. Le clown, c'est celui qui n'a pas le droit d'être là, et qu'on met à la porte. II ne peut se soumettre à aucune définition, aucune délimitation, aucune identité fixe. II est l'ouvert, l'illimité, l'indéfini, l'infini... À la question souvent posée à Catherine Germain pour savoir si le « genre » (dans l'acception sexuelle du terme) du clown peut avoir une implication sur sa façon d'être un clown, celle-ci répond :

« Je n'aime pas cette question. Je ne sais pas pourquoi, peut-être parce qu'elle est récurrente depuis quelques années où l'on voit de plus en plus

main, in Cervantes Fr., Germain C., Le Clown Arletti, vingt ans de ravissement, co-édition Magellan \& Cie / Éditions Maison, 2009, p. 126.

22 Idem., p. 13. 
de clowns en spectacle. Et qu'effectivement des femmes s'y intéressent. Pendant un temps, j'avais le sentiment qu'on était là dans un endroit où il n'y avait pas de sexe. Où l'être était tellement en question, qu'il était autant végétal que pictural, musical, animal qu'humain. En tout cas qu'il était indéfini, pour ne pas dire infini... Qu'il était important d'être une question, plutôt qu'une réponse. À la naissance d'un clown, on ne voit pas encore son sexe. Et puis le temps a passé. Moi aussi j'ai changé. Et je peux dire qu'Arletti est d'une étoffe féminine. Mais je m'attache en scène à toujours revenir à cet état premier du monde, au moment où on ne sait pas encore qui est qui, et c'est là où la relation avec le public prend sa source ${ }^{23}$. »

Figure 4: Le clown Arletti (Catherine Germain), dans Le 6ème jour.

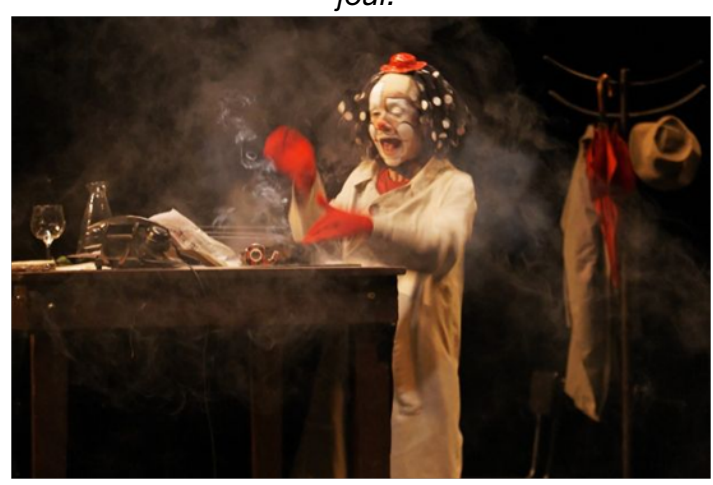

Dans l'enseignement du jeu de l'acteur, Lecoq nous a fait comprendre la place essentielle occupée par le clown. Charlot, Grock, Charlie Rivel, Albert Fratellini... sont de grands maîtres pour l'acteur, et le travail clownesque un apprentissage essentiel dans son art. Parce qu'il y a sans doute dans le clown la croyance totale, irréductible, au pouvoir de l'imaginaire. Et la naïveté sidérante du nouveau-né. Ne pas savoir, s'aventurer, se laisser posséder par ce

23 Catherine Germain, «Entretien sur Le 6ème jour avec Catherine Germain et François Cervantes ॥, propos recueillis par Stéphane Bouquet, février 2012, Théâtre contemporain.net, http://www.theatre-contemporain.net/spectacles/Le-6eme-jour/ensavoirplus/idcontent/27642, consulté le 5 janvier 2014. que l'on ne maîtrise pas, laisser la place aux manifestations d'un autre en soi, aimer le mystère de la non-personne et aimer énormément disparaître... voilà à quoi initie le clown, qui pourrait se reconnaître dans cette formule de Valère Novarina : « Le vrai acteur qui joue [entendez, le clown] aspire à rien avec autant de violence, qu'à ne pas être là.. 24

Recebido: $23 / 10 / 2017$

Aprovado: 29/01/2018
24 Novarina V., " Pour Louis de Funès ", Le Théâtre des paroles, Paris, P.O.L., 1989, p. 110. 